\title{
INCIDÊNCIA DE LESÕES MUSCULOESQUELÉTICAS ENTRE OS TRABALHADORES DOS SERVIÇOS GERAIS DO CAMPUS DOM BOSCO DA UNIVERSIDADE FEDERAL DE SÃO JOÃO DEL-REI
}

\author{
Viviane Felícia dos Reis, Felipe Rezende Ferreira Alves ${ }^{1}$, Mariana Maurício Moraes ${ }^{1}$, \\ Taynara Gabriele Aparecida de Paiva', Andréa Carmen Guimarães ${ }^{2 *}$, Luiz Gonzaga \\ Chiavegato Filho ${ }^{3} \&$ Laila Cristina Moreira Damázio ${ }^{1}$
}

REIS, V.F.; ALVES, F.R.F.; MORAES, M.M.; PAIVA, T.G.A.; GUIMARÃES, A.C.; CHIAVEGATO FILHO, L.G. \& DAMÁZIO, L.C.M. Incidência de lesões musculoesqueléticas entre os trabalhadores dos serviços gerais do campus Dom Bosco da universidade federal de São João Del-Rei. Perspectivas Online: Biológicas \& Saúde, v.10, n.34, p.33-46, 2020.

\section{RESUMO}

O presente estudo teve por objetivo avaliar a presença de Lesões por Esforços Repetitivos/Distúrbios Osteomusculares Relacionados ao Trabalho (LER/DORT) entre os funcionários dos serviços gerais do Campus Dom Bosco da Universidade Federal de São João del-Rei. Trata-se de um estudo original, com delineamento transversal, em que a amostra foi composta por 24 funcionários, os quais foram submetidos à aplicação de uma ficha de anamnese, avaliação postural e realização de testes clínico-ortopédicos específicos. Os dados foram analisados com auxílio do programa GraphPad Prism 5.0, utilizando o teste estatístico qui-quadrado, considerando o nível de significância de $5 \%$. Os resultados demonstraram que existe alta incidência de alterações posturais e queixas dolorosas entre os funcionários avaliados. Sendo que as queixas dolorosas e as alterações posturais mais encontradas foram na região da: cabeça, ombros, coluna vertebral e joelhos. Conclui-se que existe alta incidência de alterações posturais na população estudada e que torna-se necessário a realização de intervenções que possibilite minimizar esses danos.

Palavras-chaves: LER/DORT; Funcionários; Alterações posturais.

\footnotetext{
${ }^{1}$ Laboratório de Reabilitação do Departamento de Medicina (DEMED) - Universidade Federal de São João del-Rei - UFSJ Avenida Visconde do Rio Preto, Km 2, Colônia do Bengo. CEP: 36300-000 - São João del-Rei - Minas Gerais - Brasil ${ }^{2}$ Laboratório de Pesquisa e Intervenção Psicossocial - Departamento de Educação Física (DCEFS) - UFSJ

${ }^{3}$ Professor Adjunto - Departamento de Psicologia - UFSJ

(*) e-mail: andreaguimaraes@ufsj.edu.br
} 
Revista Perspectivas Online: Biológicas \& Saúde August/2020, v.10, n.34, p.33-46 ISSN: 2236-8868 (Online)

DOI: $10.25242 / 8868103420202030$

\title{
INCIDENCE OF MUSCULOSKELETAL INJURIES IN THE GENERAL SERVICES WORKERS OF DOM BOSCO CAMPUS FROM THE UNIVERSIDADE FEDERAL DE SÃO JOÃO DEL-REI
}

\author{
Viviane Felícia dos Reis, Felipe Rezende Ferreira Alves, Mariana Maurício Moraes, \\ Taynara Gabriele Aparecida de Paiva, Andréa Carmen Guimarães, Luiz, Gonzaga \\ Chiavegato Filho \& Laila Cristina Moreira Damázio
}

REIS, V.F.; ALVES, F.R.F.; MORAES, M.M.; PAIVA, T.G.A.; GUIMARÃES, A.C.; CHIAVEGATO FILHO, L.G. \& DAMÁZIO, L.C.M. Incidence of musculoskeletal injuries in the general services workers of Dom Bosco campus from the universidade federal de São João Del-Rei. Perspectivas Online: Biológicas \& Saúde, v.10, n.34, p.33-, 2020.

\begin{abstract}
The present study aimed to evaluate the presence of Repetitive Strain Injuries / Musculoskeletal Disorders at Work (RSI / WMSD) among employees of the general services of the Dom Bosco Campus of the Federal University of São João del-Rei. This is an original study, with a crosssectional design, in which the sample consisted of 24 employees, who were submitted to the application of an anamnesis form, postural assessment and specific clinical and orthopedic tests. The data were analyzed using the GraphPad

statistical test, considering the significance level of $5 \%$.

The results showed that there is a high incidence of postural changes and painful complaints among the employees evaluated. Since the most common pain complaints and postural changes were in the region of: head, shoulders, spine and knees. It is concluded that there is a high incidence of postural changes in the studied population and that it is necessary to carry out interventions to minimize these
\end{abstract} Prism 5.0 program, using the chi-square

Keywords: RSI/WMSD; Staff; Postural changes.

\footnotetext{
${ }^{1}$ Laboratório de Reabilitação do Departamento de Medicina (DEMED) - Universidade Federal de São João del-Rei - UFSJ Avenida Visconde do Rio Preto, Km 2, Colônia do Bengo. CEP: 36300-000 - São João del-Rei - Minas Gerais - Brasil ${ }^{2}$ Laboratório de Pesquisa e Intervenção Psicossocial - Departamento de Educação Física (DCEFS) - UFSJ

${ }^{3}$ Professor Adjunto - Departamento de Psicologia - UFSJ

(*) e-mail: andreaguimaraes@ufsj.edu.br
} 


\section{INTRODUÇÃO}

As lesões musculoesqueléticas são uma das doenças mais comuns relacionadas com o trabalho e, aqui no Brasil, costumam ser denominadas como lesões por esforços repetitivos (LER) ou os distúrbios osteomusculares relacionados ao trabalho (DORT). Tratam-se de uma série de doenças inflamatórias e degenerativas do sistema locomotor, que afetam predominantemente os membros superiores, mas também os membros inferiores e abrangem danos aos músculos, tendões, nervos, ligamentos, etc. $\mathrm{O}$ grau das lesões varia bastante, mas, em casos graves, podem se tornar crônicas e resultar em incapacidade e à necessidade de deixar de trabalhar (TRELHA et al., 2004; CHIAVEGATO FILHO \& PEREIRA JUNIOR, 2004). Geralmente, a ocorrência das LER/DORT está relacionada às condições de trabalho, mas especialmente à forma como o trabalho é organizado, como, por exemplo, os modelos de produção taylorista, os quais determinam estabelecimento de metas e produtividade, adequação dos trabalhadores às características organizacionais das empresas, intensificação do trabalho, aumento das jornadas, entre outros, sem levar em conta os trabalhadores e seus limites físicos e psicossociais (MINISTÉRIO DA SAÚDE, 2019).

Um levantamento feito pelo Ministério da Saúde, realizado pelos dados do Sistema de Informação de Agravos de Notificação (SINAN), mostrou que, entre 2007 e 2016, LER e DORT representaram 67.599 casos entre os trabalhadores do país (MINISTÉRIO DA SAÚDE, 2019). Algumas das principais LER/DORT que acometem os trabalhadores são as lesões no ombro e as inflamações em articulações e nos tecidos que cobrem os tendões (ARAÚJO et al., 2006). Tratam-se, de fato, de afecções multifatoriais e multidimensionais que, apesar da alta incidência ao longo das últimas décadas, não foram suficientemente comtempladas e equacionadas nas propostas de prevenção e promoção da saúde do trabalhador (WELLS, 2009).

Ocorrem principalmente devido a um desequilíbrio entre as exigências das tarefas do trabalho e as capacidades funcionais dos indivíduos, muito embora, é necessário destacar que, por exemplo, a realização de uma atividade que implica o uso de força não significa obrigatoriamente que o indivíduo desenvolverá uma lesão relacionada ao trabalho (MINISTÉRIO DA SAÚDE, 2019). O risco para o desenvolvimento de LER/DORT não depende somente da intensidade da atividade exercida, mas também de fatores psicossociais e fatores ambientais como a temperatura extrema, ruído, dimensões do posto de trabalho, vibrações, pressões mecânicas, logística de trabalho incorreta, coação para aumento da produtividade e também, posturas viciosas e inadequadas ou mantidas, carga mecânica musculoesquelético elevada e invariabilidade da tarefa (EUROPEAN AGENCY FOR SAFETY AND HEALTH AT WORK, 2012; MORAES \& BASTOS, 2013).

A atual condição de saúde do trabalhador também se inclui como um fator de risco individual e pode constituir como uma suscetibilidade acrescida para a ocorrência de LER/DORT (DA SILVA et al., 2017). Diabetes, doenças reumatológicas, certas doenças renais, antecedentes de traumatismo ou gravidez são situações de saúde que devem ser consideradas, pois predispõem o indivíduo a alterações no sistema musculoesquelético. Exemplos de fatores de risco organizacionais/psicossociais incluem: monotonia de tarefas, o que pode originar estresse; suporte social insuficiente; insatisfação com o emprego; e um modelo organizacional de produção, com pausas padronizadas, que origina situações de incompatibilidade com as capacidades do trabalhador (UVA, 2008). 
A literatura indica que profissionais que exercem atividades relacionadas à limpeza estão sujeitos a diferentes lesões musculoesqueléticas. O estudo de Kumar \& Kumar (2005) demonstra que de $36 \%$ a $56 \%$ do tempo de trabalho dos profissionais da limpeza é gasto com uma postura fletida anteriormente, $24 \%$ a $43 \%$, gasto com ambos os membros superiores elevados acima do ombro e $3 \%$ a $14 \%$, gasto com posição de cócoras. Também evidenciou-se uma alta prevalência para queixas no pescoço, ombros, cotovelo, mãos (UNGE, 2016) e região lombar (SILVA, 2003; DA SILVA et al., 2017). É possível perceber que apenas a postura adotada durante a atividade laboral já predispõe o indivíduo a distúrbios no aparelho locomotor (DA SILVA et al., 2017). Soma-se a isso outros fatores que funcionam como agravantes, como a projeção de ferramentas de limpeza que não utilizam princípios ergonômicos e de espaços de trabalho que não facilitam os processos de limpeza e solicitam o uso frequente de posturas inadequadas. Dessa forma, a limpeza é um trabalho muito árduo, que pode resultar em longos períodos de licença médica (KUMAR e KUMAR, 2006).

Diante dos diferentes fatores de risco ao desenvolvimento de lesões musculoesqueléticas entre trabalhadores da faxina e as limitações físicas, psicossociais e do trabalho que permeiam essa população, torna-se necessária investigar a incidência de LER/DORT entre esses funcionários. Busca-se verificar quais as alterações mais encontradas e propor intervenções de prevenção e promoção de saúde que possibilite melhorar a qualidade de vida e do trabalho desses profissionais dos serviços gerais do Campus Dom Bosco (CDB) da Universidade Federal de São João Del Rei/MG (UFSJ).

\section{METODOLOGIA}

\subsection{Amostra}

O estudo é do tipo original, com delineamento transversal, realizado no Laboratório de Reabilitação do Departamento de Medicina da UFSJ no período de agosto de 2018 a julho de 2019. Participaram do estudo 24 participantes, sendo 21 do sexo feminino e 3 do sexo masculino, com idade média de 43,37 anos (22-63 anos). Os trabalhadores avaliados foram profissionais da limpeza $(n=21)$ e da manutenção predial $(n=3)$. Os critérios de inclusão foram os funcionários responsáveis pelos serviços gerais na Prefeitura do CDB, sendo os profissionais da área da limpeza, eletricistas, carpinteiros, bombeiros, pedreiros e seguranças, que aceitaram participar da pesquisa e assinaram o termo de consentimento livre e esclarecido. Os critérios de exclusão foram os funcionários que realizam outros serviços além dos executados na área de serviços gerais da prefeitura do Campus, além daqueles que se recusaram a participar da pesquisa. Os participantes que aceitaram participar da pesquisa assinaram o termo de consentimento livre e esclarecido no momento da admissão na pesquisa, conforme a resolução 466/2012 do Conselho Nacional de Saúde e protocolo de análise ética CAAE: 99249118.1 .0000 .5151$.

\subsection{Procedimentos e instrumentos}

Para realização da pesquisa o projeto foi divulgado entre os servidores, e os interessados em participar agendavam uma data e horário para serem entrevistados. Foram abordados inicialmente os funcionários da limpeza, seguido dos seguranças, eletricistas, carpinteiros, pedreiros, bombeiros e seguranças. 
A avaliação foi realizada em um laboratório estruturado que possui macas e equipamentos específicos para a avaliação ortopédica e reumática do servidor. Os resultados foram obtidos através de uma ficha de anamnese semi-estruturada, elaborada pelos pesquisadores que apresentava perguntas discursivas sobre o tipo de atividade realizada, o tempo de serviço na instituição e as principais queixas osteomusculares dos participantes. Além disso, também foi usada uma ficha de avaliação postural, semiestruturada, elaborada pelos pesquisadores que apresentava informações sobre a posição das partes do corpo (cabeça, dos ombros, cotovelos, coluna vertebral, cristas ilíacas, pelve, joelhos e pés), nas visões de perfil, ântero-posterior e póstero-anterior. A escala de avaliação postural seguiu os itens de avaliação da escala de Nova Iorque, descrito no estudo de Conti et al., (2011). A avaliação postural e clínico-ortopédica foi realizada por três avaliadores que obtiveram consenso médio durante a avaliação. Os avaliadores foram treinados para realização das avaliações, além de serem orientados por uma fisioterapeuta com especialização na área e coordenadora da pesquisa.

Para avaliação clínica-ortopédica foram utilizados testes propedêuticos que possibilitaram diagnosticar diferentes complicações reumáticas e ortopédicas de acordo com a queixa inicial do funcionário. Os testes clínicos ortopédicos mais utilizados na avaliação clínico-ortopédica Hoppenfeld (2002), Dutton (2010) e Barros (2017), foram: teste de compressão e descompressão cervical, teste de Speed, Lasegue, Fabere, Adson, Phalen, Finkenstein, testes para avaliar encurtamentos musculares (músculo tenso da fáscia lata, iliopsoas, isquiotibiais, quadríceps), teste de Nachlas, Gaeslen, teste do tenista, teste do golfista, tinel de nervo ulnar e mediano. Dessa forma, a avaliação ortopédica e reumatológica foi conduzida de acordo com os sintomas apresentados pelo funcionário, sendo individualizada.

Todos os dados foram analisados com auxílio do programa estatístico GraphPad Prism 5.0, utilizando o teste estatístico qui-quadrado, considerando um nível de significância de $5 \%$. Serão analisadas as médias e frequência percentual dos dados.

\section{RESULTADOS}

A amostra foi composta por 24 participantes, sendo 21 do sexo feminino e 3 do sexo masculino, com idade média de 43,37 anos (22 - 63 anos). O tempo médio de profissão foi de 5,51 anos $(0,08$ - 20 anos $)$. As profissões encontradas entre os trabalhadores foram relacionadas à limpeza $(\mathrm{n}=21)$ e à manutenção predial $(\mathrm{n}=3)$. Ao ser questionada a escolaridade, $54 \%(\mathrm{n}=13)$ dos participantes referiu ensino fundamental I completo, $16 \%(\mathrm{n}=$ 4) referiu ensino fundamental II completo e $29 \%(n=7)$ referiu ensino médio completo.

As queixas osteomusculares relatadas estavam relacionadas à dor em $100 \%$ dos casos, sendo que, concomitantemente, também houve queixas de paresia ou de parestesia em 2 participantes. Cerca de $50 \%(\mathrm{n}=12)$ dos participantes apresentaram queixas dolorosas na coluna vertebral; 37,5\% (n=9) em membros superiores e 33\% $(n=8)$ em membros inferiores (tabela 1). 
Tabela 1. Distribuição dos sintomas dolorosos nas regiões corporais dos trabalhadores avaliados.

\begin{tabular}{lcclcc}
\hline Região Avaliada & \% & $\mathbf{N}$ & $\begin{array}{l}\text { Região } \\
\text { Avaliada }\end{array}$ & $\%$ & $\mathbf{n}$ \\
\hline Coluna Cervical & $17 \%$ & 4 & Ombros & $21 \%$ & 5 \\
Coluna Torácica & $4 \%$ & 1 & Joelho & $12,5 \%$ & 3 \\
Coluna Lombar & $33 \%$ & 8 & Pés & $8,3 \%$ & 2 \\
Perna & $4,16 \%$ & 1 & Coxa & $8,3 \%$ & 2 \\
\hline
\end{tabular}

Os testes clínico-ortopédicos foram realizados de acordo com as queixas apresentadas, sendo que alguns apresentaram mais de uma queixa. Vale salientar que, em apenas 16\% dos participantes, nenhum teste realizado apresentou-se positivo para a queixa relatada.

Com relação à avaliação postural, ao considerar as visões ântero-posterior, perfil e póstero-anterior, nenhum participante apresentou-se isento de alterações posturais. Na visão ântero-posterior, somente um participante não apresentou alterações em cabeça, ombros, cotovelo, mamilos, crista ilíaca, pelve, joelhos, pés e dedos dos pés. Dentre as regiões avaliadas, o número médio de regiões alteradas correspondeu a 3,5 (0-7). Na visão de perfil, foram avaliadas 7 regiões (cabeça, ombros, cotovelo, coluna vertebral, pelve, joelho e pés), sendo que, o número médio de regiões alteradas foi de $2,45(0-5)$. Na visão póstero-anterior, avaliou-se 8 regiões (cabeça, ombros, cotovelo, escápula, coluna vertebral, crista ilíaca, joelho e calcâneo); o número médio de regiões alteradas correspondeu a 4,45 (1-7).

$\mathrm{Na}$ visão ántero-posterior foram observadas muitas alterações posturais entre os trabalhadores, sendo que, nos membros superiores e na cabeça foram observados que $41,6 \%$ $(n=10)$ apresentaram inclinação da cabeça para a esquerda e triângulo de Talles do lado direito maior. Foi evidenciado que 33,3\% $(\mathrm{n}=8)$ apresentaram o ombro esquerdo elevado. Nos membros inferiores foram observados que $29,16 \%(n=7)$ apresentaram crista ilíaca esquerda elevada, $37,5 \%(n=9)$ com joelho valgo e 20,83\% $(n=5)$ com o joelho varo. Nos pés, $20,83 \%$ $(\mathrm{n}=5)$ com pés planos, como evidenciada na tabela 2. 
Tabela 2. Alterações posturais evidenciadas na visão àntero-posterior entre os trabalhadores avaliados.

\begin{tabular}{|c|c|c|c|c|c|}
\hline $\begin{array}{l}\text { Parâmetro Postural } \\
\text { Avaliado }\end{array}$ & $\%$ & $\mathbf{N}$ & $\begin{array}{l}\text { Parâmetro Postural } \\
\text { Avaliado }\end{array}$ & $\%$ & n \\
\hline Cabeça Inclinada para $\mathrm{E}$ & $41,6 \%$ & 10 & $\begin{array}{lll}\text { Crista } & \text { Ilíaca } & \text { Esquerda } \\
\text { Elevada } & & \end{array}$ & $\begin{array}{c}29,16 \\
\%\end{array}$ & 7 \\
\hline Cabeça Inclinada para D & $20,83 \%$ & 5 & Cristas Ilíacas Normais & $\begin{array}{c}62,5 \\
\%\end{array}$ & 15 \\
\hline Cabeça Normal & 37,55 & 9 & Pelve Normal & $\begin{array}{c}91,6 \\
\%\end{array}$ & 22 \\
\hline $\begin{array}{l}\text { Triângulo de Talles Direita } \\
\text { Maior }\end{array}$ & $41,6 \%$ & 10 & Joelho Valgo & $\begin{array}{c}37,5 \\
\%\end{array}$ & 9 \\
\hline $\begin{array}{l}\text { Triângulo de Talles Esquerda } \\
\text { Maior }\end{array}$ & $20,83 \%$ & 5 & Joelho Varo & $\begin{array}{c}20,83 \\
\%\end{array}$ & 5 \\
\hline Ombro Direito Elevado & $29,16 \%$ & 7 & Joelhos Normais & $\begin{array}{c}41,6 \\
\%\end{array}$ & 10 \\
\hline Ombro Esquerdo Elevado & $33,3 \%$ & 8 & Pés Normais & $\begin{array}{c}62,5 \\
\%\end{array}$ & 15 \\
\hline Ombros Normais & $33,3 \%$ & 8 & Pés Planos & $\begin{array}{c}20,83 \\
\%\end{array}$ & 5 \\
\hline Cotovelos sem Alterações & $33 \%$ & 8 & Dedos dos Pés Normais & $\begin{array}{c}87,5 \\
\%\end{array}$ & 21 \\
\hline
\end{tabular}

$\mathrm{Na}$ visão de perfil foram observadas na cabeça e membros superiores a presença de $37,5 \%(n=9)$ com a cabeça protrusa, e $16,7 \%(n=4)$ com os ombros protrusos. Na coluna vertebral foram observados que $25 \%(n=6)$ apresentaram hipercifose torácica, $20,83 \%(n=5)$ de hiperlordose lombar e 33,33 $(\mathrm{n}=8)$ de retificação torácica. Nos membros inferiores foram observados que $50 \%(n=12)$ apresentaram joelho recurvato, $29,16 \%(n=7)$ com a crista ilíaca esquerda elevada e pés planos (tabela 3 ). 
Tabela 3. Alterações posturais evidenciadas na visão de perfil entre os trabalhadores avaliados.

\begin{tabular}{lrrlrcr}
\hline $\begin{array}{l}\text { Parâmetro Postural } \\
\text { Avaliado }\end{array}$ & $\mathbf{\%}$ & $\mathbf{N}$ & $\begin{array}{l}\text { Parâmetro Postural } \\
\text { Avaliado }\end{array}$ & $\%$ & n \\
\hline Cabeça Protrusa & $37,5 \%$ & 9 & $\begin{array}{l}\text { Crista Ilíaca } \\
\text { Elevada }\end{array}$ & Esquerda & $29,16 \%$ & 7 \\
Ombros Protrusos & $16,7 \%$ & 4 & Retificação Torácica & $33,33 \%$ & 8 \\
Hipercifose Torácica & $25 \%$ & 6 & Joelho Recurvato & 50 & 12 \\
Hiperlordose Lombar & $20,83 \%$ & 5 & Pés Planos & $29,17 \%$ & 7 \\
\hline
\end{tabular}

$\mathrm{Na}$ região da cabeça e membros superiores foram observados que 29,2\% $(n=7)$ apresentaram cabeça inclinada para direita e $16,7 \%(n=4)$ inclinada para esquerda. O ombro direito estava elevado em 45,8\% $(n=11)$ dos trabalhadores e 20,8\% $(n=5)$ com o ombro esquerdo elevado. As escápulas elevadas em 20\% $(\mathrm{n}=5)$ dos trabalhadores. No cotovelo, $45,8 \%(n=11)$ apresentaram o triângulo de Talles do lado direito maior e $20,8 \%(n=5)$ do lado esquerdo. Na coluna vertebral foi observado que $25 \%(n=6)$ apresentaram hipercifose torácica, 33,3\% $(\mathrm{n}=8)$ hiperlordose lombar, $29,1 \%(\mathrm{n}=7)$ retificação torácica e $16,7 \%(\mathrm{n}=4)$ escoliose. Nos membros inferiores foram identificados que $41,7 \%(\mathrm{n}=10)$ apresentaram joelho valgo e $33,3 \%(n=)$ o joelho varo. As cristas ilíacas estavam elevadas em $25 \%(n=6)$ e crista ilíaca esquerda elevada em $16,7 \%(\mathrm{n}=4)$ dos avaliados. Nos pés foram observados que $54,2 \%$ $(\mathrm{n}=13)$ tiveram calcâneo valgo (tabela 4$)$. 
Tabela 4. Alterações posturais evidenciadas na visão póstero-anterior entre os trabalhadores avaliados.

\begin{tabular}{|c|c|c|c|c|c|}
\hline $\begin{array}{l}\text { Parâmetro Postural } \\
\text { Avaliado }\end{array}$ & $\%$ & $\mathbf{N}$ & Parâmetro Postural Avaliado & $\%$ & $\mathbf{n}$ \\
\hline Cabeça Inclinada para $\mathrm{E}$ & $\begin{array}{c}16,7 \\
\%\end{array}$ & 4 & $\begin{array}{l}\text { Triângulo de } \text { Talles Direito } \\
\text { Maior }\end{array}$ & $\begin{array}{c}45,8 \\
\%\end{array}$ & 11 \\
\hline Cabeça Inclinada para D & $\begin{array}{c}29,2 \\
\%\end{array}$ & 7 & $\begin{array}{l}\text { Triângulo de Talles Esquerdo } \\
\text { Maior }\end{array}$ & $\begin{array}{c}20,8 \\
\%\end{array}$ & 5 \\
\hline Cabeça Normal & $\begin{array}{c}54,2 \\
\%\end{array}$ & 13 & Joelho Valgo & $\begin{array}{c}41,7 \\
\%\end{array}$ & 10 \\
\hline Hipercifose Torácica & $25 \%$ & 6 & Joelho Varo & $\begin{array}{c}33,3 \\
\%\end{array}$ & 8 \\
\hline Hiperlordose Lombar & $\begin{array}{c}33,3 \\
\%\end{array}$ & 8 & Calcâneo Valgo & $\begin{array}{c}54,2 \\
\%\end{array}$ & 13 \\
\hline Retificação Torácica & $\begin{array}{c}29,1 \\
\%\end{array}$ & 7 & Escápulas Elevadas & $20 \%$ & 5 \\
\hline Escoliose & $\begin{array}{c}16,7 \\
\%\end{array}$ & 4 & Crista Ilíaca Elevadas & $25 \%$ & 6 \\
\hline Ombro Esquerdo Elevado & $\begin{array}{c}20,8 \\
\%\end{array}$ & 5 & Crista Ilíaca Esquerda Elevada & $\begin{array}{c}16,7 \\
\%\end{array}$ & 4 \\
\hline Ombro Direito Elevado & $\begin{array}{c}45,8 \\
\%\end{array}$ & 11 & & & \\
\hline
\end{tabular}

\section{DISCUSSÃO}

No presente estudo foi evidenciado que a maioria dos profissionais da limpeza avaliados são do gênero feminino, com idade média de 43,37 anos. Também foram evidenciadas alta prevalência de queixas dolorosas na coluna cervical, lombar (DA SILVA et al., 2017) e ombros, corroborando com os estudos de Unge (2016) e Silva (2003). No presente estudo também foi evidenciada maior prevalência de queixas dolorosas nos joelhos dos trabalhadores. Essa articulação é uma região do corpo com altas taxas de sobrecarga (DUTTON, 2010), principalmente em atividades laborais como o serviço da limpeza (KUMAR e KUMAR, 2006).

Assim, em geral foram relatadas dores na coluna, membros superiores e inferiores entre os profissionais da limpeza. Esses dados corroboram com o estudo de Pinheiro et al., (2018) onde também foram identificadas dores em todo o corpo dos profissionais da limpeza. Nesse estudo também foram citados quadros álgicos na coluna vertebral, pernas e ombros. 
Foram observadas no presente estudo várias alterações posturais em diferentes articulações. No entanto, as regiões corporais mais comprometidas foram: coluna vertebral, cervical, torácica, ombros, joelhos e pés. Essas alterações corroboram com as regiões corporais com maiores queixas dolorosas. A relação entre a posição da cabeça e a presença de dor na região cervical apresenta divergências de opiniões na literatura. Por outro lado, é sustentada a hipótese de que alterações posturais, como a protrusão da cabeça, são mais significativas em indivíduos com queixa de dor cervical quando comparados a indivíduos assintomáticos (SOARES et al, 2012).

Foram observadas alta incidência de dores nos ombros na amostra estudada e também foram evidenciadas significativas alterações posturais nos ombros desses trabalhadores. As alterações posturais nos ombros foram desnivelamento de ombros e escápulas alteradas. $\mathrm{Na}$ literatura foi mencionado que trabalhadores dos serviços gerais relacionados à faxina, que é a maioria da amostra, são submetidos a esforços intensos e tarefas de trabalho repetitivas, os quais são fatores de risco para o pescoço/ombros, bem como cotovelo/mãos (UNGE, 2016). No estudo de Dartora e Santos (2014) também foi evidenciado entre os trabalhadores de limpeza urbana maior incidência de dor no ombro e coluna vertebral e nesse estudo ainda é mencionado que a DORT afeta mais coluna cervical e membros superiores devido a sobrecarga durantes as atividades.

Um achado relativamente comum dentre os indivíduos que participaram do estudo foi a queixa dolorosa na coluna vertebral, na região cervical e lombar. Também foi evidenciada alterações posturais na coluna vertebral dos avaliados, onde foram encontradas maior incidência de alterações como: hipercifose torácica, retificação torácica, hiperlordose lombar e alterações posturais na cabeça (coluna cervical). Assim, muitos fatores podem contribuir para o desequilíbrio postural detectado entre os trabalhadores avaliados, sendo que, a atividade laboral que executam na limpeza poderá ter favorecido o surgimento ou agravamento dessas alterações, uma vez que, a permanência por muitas horas do dia em ortostatismo para a realização de suas atividades laborais favorece esse processo (KUMAR e KUMAR, 2016). No estudo de Silva et al., (2017) foi relatado que a maioria dos trabalhadores que trabalhavam em pé apresentaram sintomas dolorosos na coluna lombar e que não realizam atividade física para promover equilíbrio muscular. De acordo com Pataro (2011) a lombalgia presente em trabalhadores da limpeza urbana pode estar associada à realização dos movimentos repetitivos de flexão e rotação do tronco.

Foi evidenciado no estudo a presente de assimetria nos membros inferiores e desvios angulares nos joelhos, ou seja, a presença de joelho varo e valgo. É sabido que essas alterações podem ser congênitas. No entanto, esse alinhamento também pode ser modificado devido a outras alterações mecânicas e/ou fisiológicas, a exemplo da obesidade, pés pronados e pés planos (ALBUQUERQUE, 2012), sendo que, este último foi verificado com certa frequência nas avaliações. Outra alteração postural evidenciada com frequência no estudo foi os joelhos em recurvato. De acordo com Magee (2010) o encurtamento de isquiotibiais tem forte relação com a presença de joelhos em recurvato, corroborando com os dados coletados, uma que vez, muitos trabalhadores apresentaram encurtamento dessa musculatura.

As intervenções ergonômicas entre trabalhadores do serviço de limpeza vêm sendo empregada em alguns estudos, como de Kumar e Kumar (2006) e Monteiro et al., (2015) na tentativa de minimizar os efeitos deletérios causados pela atividade laboral desses trabalhadores. No entanto, conforme apontou Wells (2009), o conjunto das queixas e

Persp. online: biol. \& saúde, Campos dos Goytacazes, 34 (10) 33-46, 2020

https://ojs3.perspectivasonline.com.br 
alterações posturais, verificadas neste estudo, evidenciam que há muito ainda a ser feito em termos de programas de prevenção das LER/DORT e alta incidência das LER/DORT, entre os trabalhadores do Brasil, indicado pelos dados do Ministério da Saúde (2019), reforçam essa condição.

O estudo apresentou como fator limitante a dificuldade de adesão à avaliação entre os homens dos serviços gerais da instituição avaliada o que refletiu nos dados encontrados. Fica evidente que as mulheres continuam sendo mais interessadas e preocupadas com o cuidado com a saúde pessoal e isso foi verificado nesse trabalho.

\section{CONCLUSÃO}

Conclui-se que, dentre os trabalhadores avaliados a sua maioria é do serviço de limpeza, do gênero feminino e com idade média de 43 anos. As regiões corporais que apresentaram maiores queixas dolorosas e alterações posturais foram: coluna vertebral (cervical, torácica e lombar), nos ombros e joelhos. Esses dados demonstram a necessidade de intervenções de prevenção nos ambientes de trabalho desse setor, como forma de minimizar os impactos deletérios causados pela atividade laboral da limpeza.

Dessa forma, por tratar-se de um problema multidimensional, recomenda-se a realização de uma intervenção ergonômica, além de uma intervenção fisioterapêutica com os participantes do estudo, a fim de diminuir a incidência de queixas osteomusculares no setor e, portanto, proporcionar melhor qualidade de vida e disposição para a prática de atividades laborais. Para além desse estudo, é preciso pensar também novas formas de organização de trabalho, de modo a criar um equilíbrio entre as exigências das tarefas do trabalho e as capacidades funcionais dos indivíduos.

\section{REFERÊNCIAS}

ALBUQUERQUE, R.; BARRETTO, J.; CARVALHO, A.; PINHEIRO, V.; MERCANTE, B.; \& ASSIS, D. Análise comparativa da mensuração do eixo anatómico do joelho. Revista Portuguesa de Ortopedia e Traumatologia, 20(1): 81-86, 2012.

ANDRADE, C. B.; \& MONTEIRO, M. I. Envelhecimento e capacidade para o trabalho dos trabalhadores de higiene e limpeza hospitalar. Revista da Escola de Enfermagem da USP, v. 41, n. 2, p. 237-244, 2007.

ARAÚJO, E.C.L.S.; VIDOTTI, E.; SANTOS, C.F.; DE ALCÂNTARA FERREIRA, T.R.; PEREIRA, R.S.; \& MEDINA, A.B.P. Avaliação de qualidade de vida de pacientes com LER/DORT atendidos pelo Sistema Único de Saúde (SUS). UNOPAR Cient., Ciênc. Biol. Saúde. Londrina. v.8, n.1, p.39-43, 2006.

BARROS, G.M.T. Ortopedia e Traumatologia. Editora Elsevier. Sociedade Brasileira de Ortopedia e Traumatologia. 2017.

BRASIL. Ministério da Saúde. Secretaria de Vigilância em Saúde. Departamento de Vigilância de Doenças e Agravos não Transmissíveis e Promoção da Saúde. Saúde Brasil 
2018 uma análise de situação de saúde e das doenças e agravos crônicos: desafios e perspectivas. Brasília: Ministério da Saúde, 2019. Disponível em: $<$ http://bvsms.saude.gov.br/bvs/publicacoes/saude_brasil_2018_analise_situacao_saude_doe ncas_agravos_cronicos_desafios_perspectivas.pdf``. Acesso em 21/03/2020.

BRASIL. Ministério da Saúde. Secretaria de Vigilância em Saúde. Departamento de Vigilância em Saúde Ambiental e Saúde do Trabalhador. Dor relacionada ao trabalho: lesões por esforços repetitivos (LER): distúrbios osteomusculares relacionados ao trabalho (Dort). Brasília: Editora do Ministério da Saúde, 2012. Disponível em: $<$ http://bvsms.saude.gov.br/bvs/publicacoes/dor_relacionada_trabalho_ler_dort.pdf $>$. Acesso em: 21/03/2020.

CHAVES, P.J.; OLIVEIRA, F.E.M.; \& DAMÁZIO, L.C.M. Incidence of Postural Changes and Temporomandibular Disorders in Students. Acta Ortopédica Brasileira, v. 25, n. 4, p. 162-164, 2017.

CHIAVEGATO FILHO, L.G.; PEREIRA JR., A. LER / DORT: multifatorialidade etiológica e modelos explicativos. Interface, v. 8, n. 14, p. 149-162, 2004.

COSTA, J.C.; GUEDES, J.; BAPTISTA, J.S.; VAZ, M.; STYLIANO, M.M.; \& PINHO, A.R. Evaluation of the Methodological Criteria Used in Studies About the Assignment of Musculoskeletal Injury (disease) at Work. A Systematic Review. Occupational Safety and Hygiene - SHO 2012 Sociedade Portuguesa de Segurança e Higiene Ocupacionais.

CONTI, P.B.M.; SAKANO, E.; RIBEIRO, A.G.O.; SCHIVINSKI, C.I.S.; RIBEIRO, J.D. Avaliação da postura corporal em crianças e adolescentes respiradores orais. J. Pediatr., v.87, n.4, p.357-363, 2011.

DA SILVA, R.K.; RECKZIEGEL, M.B.; BURGOS, M.S.; POHL, H.H. Dor lombar e sua relação com a flexibilidade e os desvios posturais em trabalhadores rurais de municípios da microrregião sul do Vale do Rio Pardo/RS. Revista Fisioterapia Brasil, v.18, n.2, p.130-139, 2017.

DARTORA, J.; \& SANTOS, M.V. Cinesioterapia laboral preparatória para trabalhadores de urbanização de uma empresa do Vale do Taquari/RS. Revista Destaques Acadêmicos, v.6, n.3, 2014.

DUTTON, MARK. Fisioterapia Ortopédica: Exame, Avaliação e Intervenção. 2 ed. Editora Artmed, 2010.

EUROPEAN AGENCY FOR SAFETY AND HEALTH AT WORK (EASHW). Musculoskeletal disorders in agriculture. Bilbao: EASHW; 2012.

FALCÃO, F.R.C.; \& MARINHO, A.P.S.; \& SÁ, K.N. Correlação dos desvios posturais com dores músculo-esqueléticas. Revista de Ciências Médicas e Biológicas, v. 6, n. 1, p. 54-62, 2007.

FEUERSTEIN, M. SHAW, W.S.; NICHOLAS, R.A.; \& HUANG, G.D. From confounders to suspected risk factors: psychosocial factors and work-related upper extremity disorders. Journal of Electromyography and Kinesiolog, v. 14, n. 1, p. 171-178, 2004.

KUMAR, R.; \& KUMAR, S. Musculoskeletal risk factors in cleaning occupation - A literature review. International Journal of Industrial Ergonomics, Elsevier, 2006.

MAGEE, D.J. Avaliação Musculoesquelética. 5a ed. Barueri, SP: Manole, 2010. 
MIRANDA, L.C.; CARNIDE, F.; \& LOPES, M.F. Lesões musculoesqueléticas relacionadas com o trabalho: Magnitude do problema a nível nacional. Disponível em: https://www.dgs.pt/ficheiros-de-upload-3/pncdr- proj_relatorio- lmert-pdf.aspx. Acesso em: 10/03/2018.

MONTEIRO, J.; LYRIO, A.; GOMES, C.; RANGEL, G.; \& SILVA, L. Benefícios da ginástica laboral como medida de prevenção para doenças ocupacionais: um estudo sob a ótica da ergonomia. Biológicas \& Saúde v. 5, n. 18, 2015.

MORAES, P. W. T., \& BASTOS, A. V. B. As LER/DORT e os fatores psicossociais. Arquivos Brasileiros de Psicologia, v. 65, n. 1, p. 1-19, 2013.

PARATO, S.M.S. Lombalgia em trabalhadores de limpeza urbana. 2011. 154f.

Dissertação (Mestrado em Saúde, Ambiente e Trabalho) - Faculdade de Medicina da Bahia da Universidade Federal da Bahia.

PINHEIRO, L.M.; STIEHL, A; L., JAHN, G.F.; VEIGA, A.M., TOLENTINO, G.S.; STURMER, G. Presença de dores e desconfortos osteomioarticulares em trabalhadores do setor de limpeza predial de uma instituição de ensino superior. XXIII Seminário Interinstitucional de Ensino, Pesquisa e Extensão-Ciência e Diversidade. Out., 2018.

SANTOS, D.M. LER/DORT atinge 3,5 milhões de trabalhadores. Disponível em: noticia/2016/2/pesquisadores-da-fundacentro-comentam-sobre-a-lerdort. Acesso

em: 21/03/2018.

SERRANHEIRA, F. UVA, A.S.; \& ESPÍRITO-SANTO, J. Estratégia de Avaliação de lesões músculo-esqueléticas de membros superiores ligadas ao trabalho aplicada na indústria de abate de desmancha de carne em Portugal. Rev. Brasileira de Saúde Ocupacional, vol. 34, nº 119, São Paulo, Jan./June 2009.

SILVA, R.K.; RECKZIEGEL, M.B.; BURGOS, M.S.; POHL, H.H. Dor lombar e sua relação com a flexibilidade e os desvios posturais em trabalhadores rurais de municípios da microrregião sul do Vale do Rio Pardo/RS. Revista Fisioterapia Brasil, v.18, n.2, p. 130139, 2017.

SILVA, L.H. Características pessoais e organizacionais relacionadas a lombalgia em faxineiras. Universidade Federal de Santa Catarina, Programa de Pós- Graduação em Engenharia de Produção - Ergonomia, 2003.

SOARES, J.C.; WEBER, P.; TREVISAN, M.E.; TREVISAN, C.M.; \& ROSSI, A.G. Correlação entre postura da cabeça, intensidade da dor e índice de incapacidade cervical em mulheres com queixa de dor cervical. Revista Fisioterapia e Pesquisa, v. 19, n. 1. São Paulo: jan./mar. 2012.

TRELHA, C.S.; GUTIERREZ, P.R.; MATSUO, T. Prevalência de sintomas músculoesqueléticos em fisioterapeutas da cidade de Londrina. Fisioterapia \& Pesquisa. v.11, n.1, 2004.

UNGE, Jeannette. Exposure, musculoskeletal disorders and organizational aspects of hospital cleaning work. Lund University, Faculty of Medicine, 2016.

UVA, A.; CARNIDE, F.; SERRANHEIRA, F.; LOPES, M. F.; \& MIRANDA, L. Ministério da Saúde. Direcção-Geral da Saúde. Direcção de Serviços de Cuidados de Saúde. Guia de orientação para prevenção das lesões musculoesqueléticas e relacionadas com o trabalho: programa nacional contra as doenças reumáticas. Lisboa, Portugal: DGS, 2008.

Persp. online: biol. \& saúde, Campos dos Goytacazes, 34 (10) 33-46, 2020 
WALSH, I.A.P.D.; CORRAL, S.; FRANCO, R.N.; CANETTI, E.E.F.; ALEM, M.E.R.; \& COURY, H.J.C. Capacidade para o trabalho em indivíduos com lesões músculo-esqueléticas crônicas. Revista de Saúde Pública, v. 38, p. 149-156, 2004.

WELLS, Richard. Why have we not solved the MSD problem? Work, v. 34, n. 1, 117-121, 2009.

WORLD HEALTH ORGANIZATION. Global Recommendations on Physical Activity for Health. Disponível em: <https://www.who.int/dietphysicalactivity/physical-activityrecommendations-5-17years.pdf $>$. Acesso em 18/08/2019. 